\title{
Kinetic Physical Etching for Versatile Novel Design of Well Ordered Self-Affine Nanogrooves
}

\author{
Sebastiaan van Dijken, Dennis de Bruin, and Bene Poelsema* \\ MESA+ Research Institute and Faculty of Applied Physics, University of Twente, \\ P.O. Box 217, 7500 AE Enschede, The Netherlands
}

(Received 8 December 2000)

\begin{abstract}
Ion bombardment at extreme grazing incidence leads to the formation of remarkably well ordered, one to two atomic layer deep, parallel grooves on a $\mathrm{Cu}(001)$ surface. These self-organized grooves are oriented parallel to the ion's plane of incidence and their period can be controlled between $\sim 4$ and $15 \mathrm{~nm}$. We have identified two distinct temperature regimes: Between about 200 and $300 \mathrm{~K}$ the substrate temperature controls the period, while below 200 to about $150 \mathrm{~K}$ the ion energy does. The groove separation distribution shows distinct self-affine character. The physical origin of the novel nanostructures is discussed.
\end{abstract}

Systematic investigation of the physical behavior of structures in reduced dimensions requires the controlled preparation of well-defined nanometer features on surfaces. Recently, great progress has been achieved in this area. Advanced contact imprinting techniques are currently applied with great success [1-3]. However, there still is a strong need for versatile and reversible manufacturing of structures at length scales well below $20 \mathrm{~nm}$, opening new avenues for thorough investigation of quantum physical properties of metallic nanostructures. One possible route is the construction of individual structures by applying scanning probe based atom manipulation techniques [4,5]. Although these methods are highly attractive for looking at the level of individual structures, they bear the disadvantage that they are quite time consuming and rely for their characterization on sometimes intrusive highresolution probes. Powerful alternative avenues are provided by thermodynamic and kinetic self-organization, allowing the evolution of nanostructures with well-defined sizes and homogeneously distributed within macroscopic areas. Thermodynamic self-organization typically deals with the relaxation of strain on surfaces or in thin films, e.g., Refs. [6-8]. This route couples the advantage of narrow size and distance distributions with drawbacks on independent variation of the structure size and separation. Kinetic self-organization during growth or etching does not suffer from this shortcoming: separation and size can be varied independently. Usually, however, such structures suffer from insufficiently narrow size and separation distributions. Quite narrow size distributions of metal nanoparticles have already been achieved through initial growth on strained films with ordered dislocation networks [9]. Another possible pathway for obtaining improved size distributions is provided by molecular beam deposition at grazing incidence, which leads to the formation of ordered parallel ripples [10,11]. However, these methods suffer from ill-defined vertical terrace distributions. This applies also for off-normal $\left(70^{\circ}\right)$ ion bombardment induced stripe structures on typically nonisotropic surfaces $[12,13]$. In the successful process of searching for novel and versatile means to prepare macroscopically homogeneous surfaces we have encountered an unanticipated new physical phenomenon: Grazing incidence ion beam etching leads to the formation of self-affine, highly ordered arrays of parallel, shallow grooves. Control of their mutual distance in a relevant range of $4-15 \mathrm{~nm}$ is gained by variation of the substrate temperature and of the incident ion energy.

In this study we are particularly interested in ensemble properties of the novel phenomenon, such as average separation between the grooves and its standard deviation. Our experimental method of choice is high-resolution electron diffraction (HR- or SPA-LEED). This technique directly provides the wanted information without heavy averaging and possible bias. The experiments have been conducted in ultrahigh vacuum (base pressure $<10^{-10} \mathrm{mbar}$ ). The substrate temperature is always quenched to about $100 \mathrm{~K}$ immediately after switching off the etching ion beam, to minimize possibly disturbing annealing.

The investigated system is a $\mathrm{Cu}(001)$ surface which, before each new experiment, has been prepared with average terrace widths exceeding $100 \mathrm{~nm}$. The face-centered cubic (001) surface was chosen because of its fully isotropic diffusion behavior.

When bombarding $\mathrm{Cu}(001)$ with a monochromatic ion beam of about $1 \mathrm{keV}$, at grazing incidence $\left(\sim 80^{\circ}\right.$ from the normal), extremely well ordered arrays of parallel grooves are formed. The orientation of the grooves is parallel to the plane of incidence of the ion beam. A characteristic example of SPA-LEED data is given in Fig. 1. The data have been taken after bombarding the $\mathrm{Cu}(001)$ surface with $800 \mathrm{eV} \mathrm{Ar}^{+}$ions, incident at a polar angle of $80^{\circ}$ and along a $\langle 110\rangle$ azimuth. The surface temperature was set at $250 \mathrm{~K}$ and the ion flux and fluence were $5 \times 10^{16}$ ions $\mathrm{m}^{-2} \mathrm{~s}^{-1}$ and $1.8 \times 10^{20}$ ions $\mathrm{m}^{-2}$ (about 12 ions/surface atom), respectively. Under these conditions only a few monolayers are eroded. The sputter yield amounts to less than one atom 


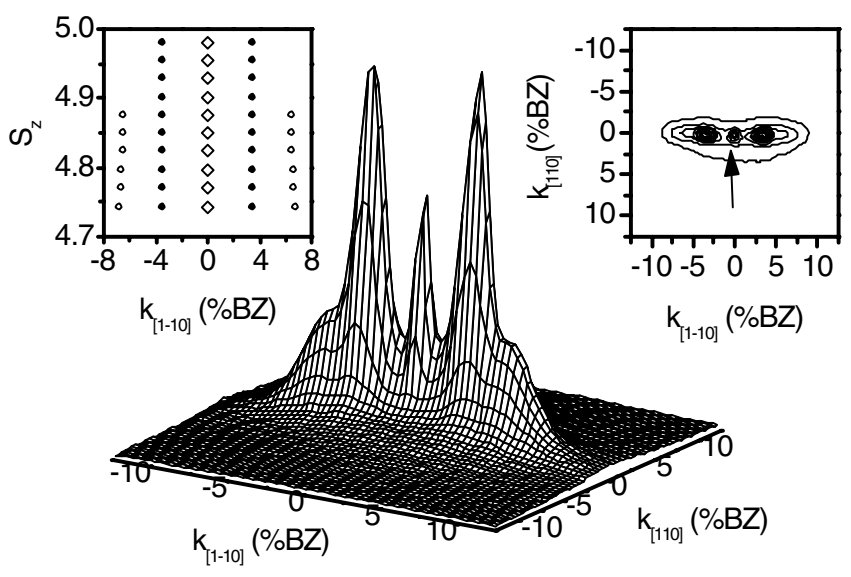

FIG. 1. High-resolution low-energy electron diffraction profile of the specular beam and contour plot (right inset). The data have been taken after bombarding the $\mathrm{Cu}(001)$ surface with $800 \mathrm{eV} \mathrm{Ar}^{+}$ions, incident at $80^{\circ}$ from the surface normal, along the [110] azimuth. The surface temperature was set at $250 \mathrm{~K}$ and the ion flux and - dose were $5 \times 10^{16}$ ions $\mathrm{m}^{-2} \mathrm{~s}^{-1}$ and $1.8 \times$ $10^{20}$ ions $\mathrm{m}^{-2}$, respectively. The profile was taken at $273 \mathrm{eV}$ $\left(S_{z}=4.88\right)$. The left inset shows the position of the first and second order separation peaks of the grooves as a function of the phase-factor $S_{z}$.

per ion at normal incidence and the geometry is deliberately set to meet near total reflection conditions [14]. The data in Fig. 1 illuminate many noteworthy facts as follows.

(i) The diffraction profile is essentially one-dimensional with a central Bragg peak and two side peaks, indicating the emergence of grooves, oriented parallel to the plane of incidence of the ions (right inset).

(ii) The position of the side peaks does not depend on the change of the perpendicular scattering vector $S_{z}$ (left inset). $S_{z}$ is defined as the path length difference for scattering from two adjacent layers in units of $2 \pi$ over the wavelength. Therefore, they are not related to facets [15], but rather disclose that the grooves are composed of very shallow structures.

(iii) The in-plane widths of the diffraction peaks reveal that the average length of the grooves amounts to as much as $22 \mathrm{~nm}$ [15].

(iv) The appearance of clearly developed humps at the expected positions of the second order peak demonstrates a surprisingly high degree of order perpendicular to the grooves. Such high order has never been observed in selforganization features in growth studies [16-20].

We note that striped patterns develop also upon ion etching at polar angles larger than $85^{\circ}$ along the $\langle 100\rangle$ and $\langle 310\rangle$ azimuths. The highest order, however, is obtained along $\langle 110\rangle$ (Fig. 1).

The nanogrooves appear reversibly under grazing incidence ion etching, following a sample annealing step, in which the initial distance between steps of about $100 \mathrm{~nm}$ is restored. Variation of the substrate temperature gives rise to different periodicity of the grooves. The data in Fig. 2

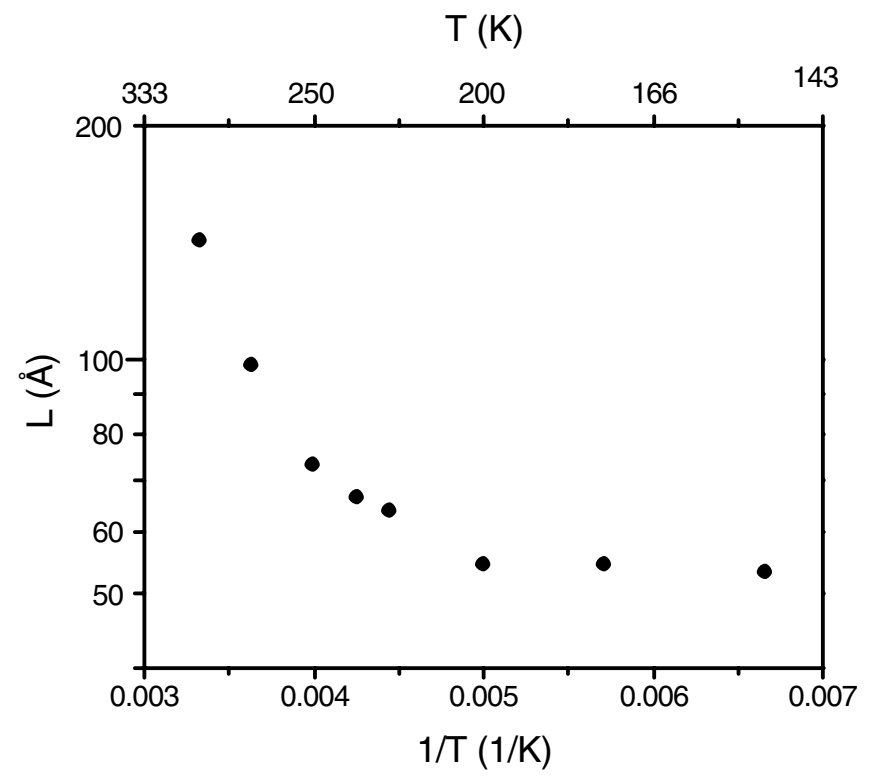

FIG. 2. Average groove separation as a function of substrate temperature. The remaining physical conditions, such as geometry, ion flux, and - dose, are identical to those in Fig. 1. The data have been taken at $100 \mathrm{~K}$ to avoid annealing after switching off the ion beam.

show the average distance between the parallel grooves as a function of temperature. The physical conditions (geometry, ion flux, and ion fluence) are identical to those in Fig. 1. Figure 2 clearly demonstrates the variation of the period of the grooves between about 4 and $15 \mathrm{~nm}$. The parallel grooves appear in a wide temperature window, between about 150 and $300 \mathrm{~K}$. The limitation at high temperatures is set by the competition between the coarsening rate, governed by various (step, kink, adatom, etc.) free energies of the $\mathrm{Cu}(001)$ surface and the etching rate set by the incident ions, depending on geometry, ion energy, etc. The low temperature side is determined by freezing-in thermally activated diffusion of adatoms along $\langle 110\rangle$ step edges [21].

The data of Fig. 2 show two distinct temperature regimes. In regime I, below $200 \mathrm{~K}$, the average distance between the grooves is temperature independent. In regime II, above $200 \mathrm{~K}$, the groove separation increases with temperature. This increase is related to increasing mobility of ion-induced vacancies. The slope of the line through the "high" temperature data points seems to indicate a thermally activated diffusion process with an activation energy of about $0.3 \mathrm{eV}$. This compares rather well to values calculated for vacancy diffusion on $\mathrm{Cu}(001)[21,22]$.

Figure 3 shows scaled specular peak profiles obtained after grazing incidence ion etching at three substrate temperatures within regime II. The data have been obtained after deconvolution with the instrument response function, approximated with a spline function, being the origin of the small wiggles in the data. The central Bragg peaks have been omitted. The side peaks have been scaled with one 


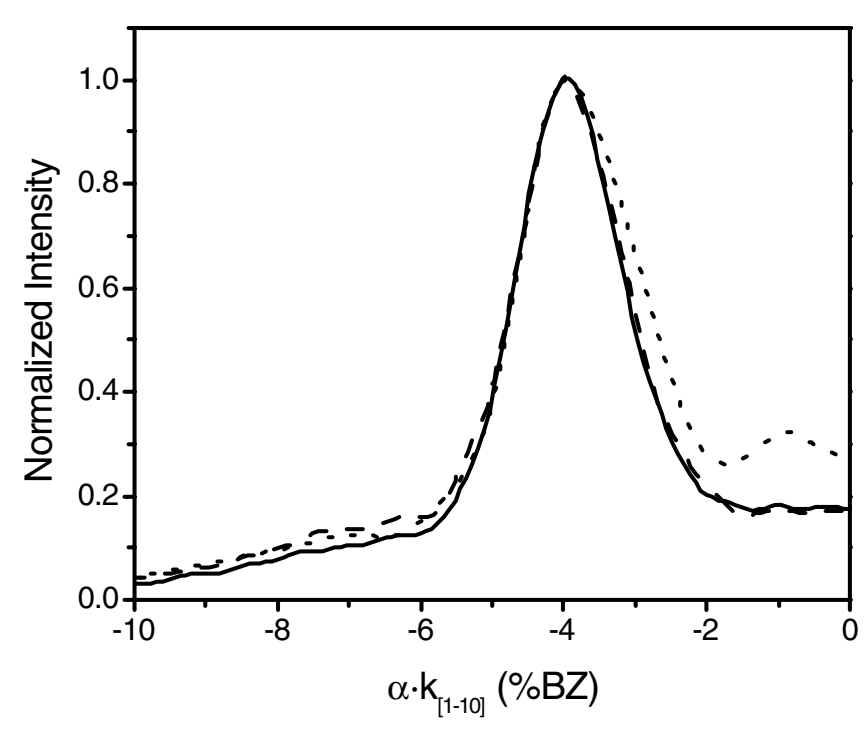

FIG. 3. Normalized specular peak profiles (see text for details); data obtained at $225 \mathrm{~K}$ (full), $250 \mathrm{~K}$ (dashed), and $300 \mathrm{~K}$ (dotted) after deconvolution with the instrument response function.

factor, being the parallel change of the wave vector at the maximum of the side peaks. To very good approximation the shapes of the side peaks are identical. This relevant fact evidences identical shapes and thus scaling behavior of the groove separation distribution function. The periodic groove patterns show distinct self-affine behavior of the periodic and linear grooves. This relevant fact reveals that thermodynamics does not play a significant role. Assuming thermodynamic governing the step pattern would imply that the width of the step separation distribution should be temperature dependent, related to the step dynamics and entropic repulsion [23]. One should expect the standard deviation to vary by about a factor of 1.5 using kink-formation energies of $0.125 \mathrm{eV}$ [24] and assuming a repulsive step-step interaction roughly proportional to their squared inverse distance. Thus self-affinity reveals the pure kinetic nature of the groove evolution. This is indicative of a dominant contribution to the formation of the periodic groove pattern by a single thermally activated process, most probably the diffusion of monovacancies.

The width of the side peaks (Fig. 3) allows estimating the standard deviation of the groove separation distribution function. It amounts to about $20 \%$, being much smaller than typical values obtained during growth and etching around normal incidence. This fact is consistent with the presence of second order diffraction features in Fig. 3. These were never observed in peak profiles measured after growth or ion bombardment after normal incidence.

The scenario for the evolution of the regular arrays of parallel linear and shallow grooves must include the following ingredients: (1) the separation between the grooves is diffusion controlled with an activation energy of roughly $0.3 \mathrm{eV}$; (2) the grooves develop even in the absence of thermally activated adatom (or vacancy) mobility; (3) ther- mally activated ledge atom diffusion is crucial; and finally (4) the novel structures do not develop when the incident ion beam conditions (mass, energy, polar and azimuthal angles of incidence) allow significant erosion of terraces. A plausible scenario goes as follows: Isolated vacancies are formed upon ion impact. (Simulations [14] have shown that this is a highly infrequent process on terraces under the given conditions: Most of the incident ions reflect specular.) Adatoms, possibly created through the same impact [25], or diffusing around after being generated previously, may annihilate the freshly produced monovacancy. The surviving vacancies diffuse and will meet others to form stable nuclei [26]. Since diffusion on $\mathrm{Cu}(001)$ is intrinsically isotropic (e.g., [19]), there is no preference for finding neighboring clusters along specific azimuth directions. Once a stable vacancy cluster is formed, it grows further in a strongly anisotropic way since illuminated ascending steps are eroded with high preference for two reasons: First, they are not screened from the ions by neighbors and, second, the incident ion flux is strongly enhanced for geometric reasons. As a result, the vacancy clusters become elongated along the plane of incidence and they coalesce first in this direction. Immediately after coalescence the edges of the merged clusters are smoothened efficiently, again for two reasons. First, thermodynamics tends to reduce a possible hyperthermal density of kinks, facilitated by the high mobility of ledge adatoms along $\langle 110\rangle$ steps on this fcc(001) surface. Second, illuminated kink atoms are eroded with high preference (see above). Note that the equilibrium shape of the vacancy islands is a square with [110]-oriented step edges. Striving for equilibrium shapes counteracts the kinetic preference for elongated clusters and dominates at sufficiently high diffusion rates, giving rise to the high temperature limes discussed above. The low temperature limes is set by the restricted [110]-ledge adatom diffusion.

The high degree of ordering is related to the fact that vacancies formed in the remaining terraces, bordering the linear vacancy clusters, have a very high probability to diffuse to the descending steps and get incorporated in these ledges. When for statistical reasons a relatively wide terrace remains, the descending step edge is eroded fast. In reverse, narrow terraces give rise to slow erosion. As a result, the centers of gravity of the linear vacancy clusters shift, resulting in higher order. The centers of gravity of nearby vacancy clusters move away from each other, while those of distant ones move together. Thus the periodicity of the grooves improves, being further enhanced by memory effects [27,28].

Finally we have studied in more detail another intriguing novel behavior (temperature regime I). We emphasize that the observed temperature independent period of the grooves depends neither on ion flux nor on ion fluence. However, as illustrated by the data in Fig. 4 the period does depend on the energy of the incident ions. Variation of the projectile's energy between 200 and $2000 \mathrm{eV}$ allows 


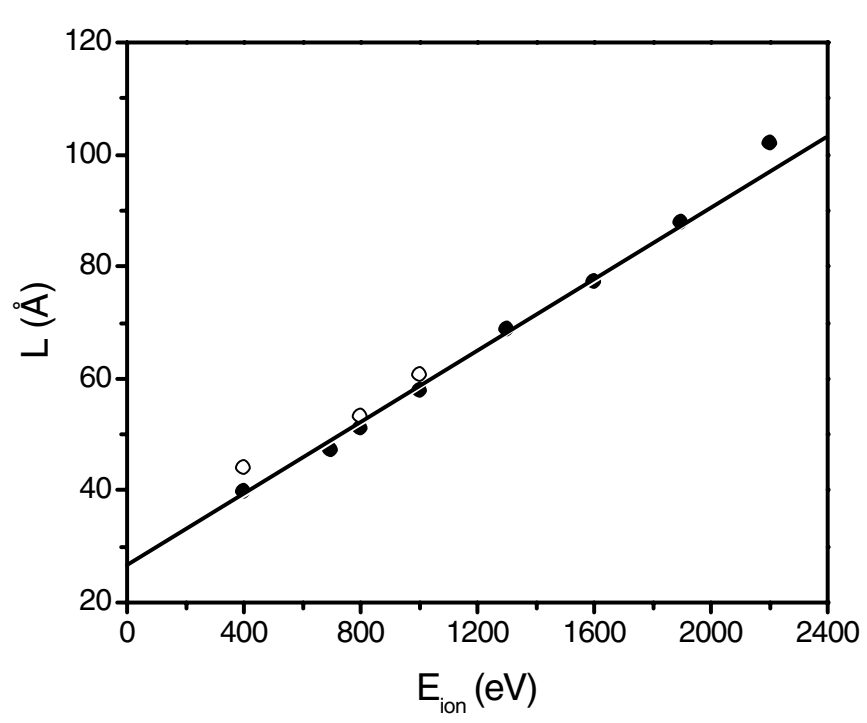

FIG. 4. Groove wavelength as a function of the ion energy at $150 \mathrm{~K}$. The ions $\mathrm{Ar}^{+}$and $\mathrm{Ne}^{+}$were incident at $80^{\circ}$ from the surface normal, along the [110] azimuth.

varying the average groove separation between about 4 to $10 \mathrm{~nm}$. The present data are probably the most basic example of irradiation-assisted diffusion as observed in early days $[29,30]$. The remarkable feature is rationalized in terms of an ion impact induced "hot spot." A finite time is required for the leakage of the energy transferred by the impinging ion towards macroscopically distant places [31]. A typical time scale is in the order of nanoseconds [32]. The transient hot spot emerges exactly near the position, where the monovacancy is generated. Within the finite lifetime of the hot spot the freshly created vacancy can diffuse, meet others, and form a stable nucleus. In all cases thermal diffusion of ledge adatoms remains active. Figure 4 reveals a linear relationship between groove period and ion energy. We have presently no explanation for this remarkable feature. At higher energies one should expect deviations from this behavior since the ion energy is then distributed in deeper areas of the crystal. In fact, we have observed deviations for $2 \mathrm{keV} \mathrm{Ne}$ ions. Note $^{+}$ that the intersection with the ordinate occurs at a length of about $2.5 \mathrm{~nm}$. This value nicely corresponds to the minimum attainable length scale during growth [19], which is attributed to ledge adatom mobility on $\mathrm{Cu}(001)$ [33].

To summarize, extreme grazing incidence ion bombardment leads to the unanticipated formation of well-defined, parallel linear nanogrooves. For $\mathrm{Cu}(001)$ we have identified two distinct temperature regimes. For $800 \mathrm{eV}$ ions, above $200 \mathrm{~K}$ the temperature governs the period of the grooves, while below $200 \mathrm{~K}$ the ion energy does. At low temperature, ions creating vacancies cause a transient hot spot, allowing nonthermal mobility. The lowest temperature [for $\mathrm{Cu}(001)$ about $150 \mathrm{~K}$ ] is determined by finite [110]-ledge adatom mobility. The high temperature limes are set by thermodynamics striving for square equilibrium shaped vacancy clusters. The nanogrooves, emerging in the later regime, show distinct self-affine behavior.

We thank Dr. Van de Waal, Dr. Van Silfhout, Dr. Wormeester, and Dr. Zandvliet for critical reading of the manuscript and Dr. Wormeester for his assistance in the deconvolution of the peak profiles.

*Corresponding author.

Email address: b.poelsema@tn.utwente.nl

[1] J. R. Jackman et al., Science 269, 664 (1995).

[2] S.-Y. Chou et al., Science 272, 85 (1996).

[3] T. I. Kemins et al., Appl. Phys. Lett. 74, 1773 (1999).

[4] D. M. Eigler and E. K. Schweizer, Nature (London) 344, 524 (1990).

[5] G. Meyer et al., Appl. Phys. A 60, 343 (1995).

[6] K. Kern et al., Phys. Rev. Lett. 67, 855 (1991).

[7] P. Zeppenfeld et al., Phys. Rev. Lett. 72, 2737 (1994).

[8] H. J. W. Zandvliet et al., Phys. Rev. Lett. 75, 3890 (1995).

[9] K. Bromann et al., Eur. Phys. J. 9, 25 (2000).

[10] S. van Dijken et al., Phys. Rev. Lett. 82, 4038 (1999).

[11] S. van Dijken et al., Phys. Rev. B 61, 14047 (2000).

[12] S. Rusponi et al., Appl. Phys. Lett. 75, 3318 (1999).

[13] S. Rusponi et al., Phys. Rev. Lett. 81, 2735 (2000).

[14] B. Poelsema et al., Surf. Sci. 64, 537 (1977).

[15] S. van Dijken, Ph.D. thesis, University of Twente, 2000.

[16] J.-K. Zuo et al., Phys. Rev. Lett. 78, 2791 (1997).

[17] H. Dürr et al., Surf. Sci. Lett. 328, L527 (1995).

[18] H.-J. Ernst et al., Phys. Rev. Lett. 72, 112 (1994).

[19] L. C. Jorritsma et al., Phys. Rev. Lett. 78, 911 (1997).

[20] M. Horn-von Hoegen, Z. Kristallogr. 214, 1 (1999).

[21] C.-L. Liu, Surf. Sci. 316, 294 (1994).

[22] G. Boisvert et al., Phys. Rev. B 58, 12667 (1998).

[23] E. D. Williams and N. C. Bartelt, Science 251, 393 (1991).

[24] G. Schulze Icking-Konert et al., Phys. Rev. Lett. 83, 3880 (1999).

[25] T. Michely and C. Teichert, Phys. Rev. B 50, 11156 (1994).

[26] J. A. Venables, Philos. Mag. 27, 697 (1978).

[27] B. Poelsema et al., Appl. Phys. A 53, 369 (1991).

[28] G. Rosenfeld et al., Phys. Rev. Lett. 71, 895 (1993).

[29] J. Y. Cavaillé and M. Drechsler, Surf. Sci. 75, 342 (1978).

[30] M. Drechsler et al., Surf. Sci. 97, 111 (1980).

[31] H.-J. Ernst et al., Science 279, 679 (1998).

[32] James Sprague (private communication).

[33] M. Breeman et al., Phys. Rev. B 54, 16440 (1996). 